\title{
Therapeutic effect of YSY01 on osteoarthritis in rabbit: involvement of suppressing signaling pathway of NF-kB and MMP-9
}

\section{Type}

Research paper

\section{Keywords}

osteoarthritis, Apoptosis, MMP-9, NF-kB, YSY01A

\begin{abstract}
Introduction

It has been reported that the NF-KB and MMP-9 signaling pathways were involved in the pathogenesis of osteoarthritis $(O A)$, while the treatment of OA by YSY01 could inhibit the proteasome activity. Therefore, we aimed to study the therapeutic effect of YSY01A treatment on OA via interfering with expression of NF-KB and MMP-9.

\section{Material and methods}

Western blot analysis and immunohistochemistry (IHC) assays were used to measure the expression of NF-KB and MMP-9 in animal models established via SH treatment or cellular models established via sodium nitroprusside (SNP) treatment. MTT assay and flow cytometry analysis were performed to observe the effect of YSY01 treatment on cell viability and apoptosis.
\end{abstract}

\section{Results}

The decreased expression of NF-KB and MMP-9 was observed in OA rabbits and cells treated by YSY01A, thus indicating the inhibitory effect of YSY01A on NF-KB and MMP-9 expression. And YSY01A exhibited a positive therapeutic effect on OA both in vivo and in vitro by inhibiting the expression of NF-KB and MMP-9. Meanwhile, YSY01A treatment could increase cell viability to a certain degree and decrease the apoptosis index, which suggested the application of YSY01A in OA therapy.

\section{Conclusions}

The levels of NF-KB and MMP-9 which were associated with aggravated apoptosis were upregulated in OA models in vivo and in vitro. YSY01A treatment could down-regulate the expression of NF-KB and MMP-9 and inhibit cell apoptosis, thus reducing the severity of OA. 
Therapeutic effect of YSY01 on osteoarthritis in rabbit: involvement of suppressing signaling pathway of NF-kB and MMP-9

Zhou Yang ${ }^{1}$, Feng Zhan ${ }^{1 *}$, Shu-dian Lin ${ }^{1}$, Ying Liu ${ }^{1}$, Yu-wei Zhan ${ }^{1}$, Jiao-mei Cheng ${ }^{2}$

1. Department of Rheumatology \& Immunology, Hainan General Hospital, Haikou 570000, P.R. China

2. Department of Rheumatology \& Immunology, Xiangya Hospital, Central South University, Changsha 410008, P.R. China

*Correspondence to Feng Zhan, Department of Rheumatology \& Immunology, Hainan General Hospital, No.19 Xiuhua Road, Xiuying District, Haikou, Hainan Province, P.R. China

Email: bonescience@163.com

Tel: +86-0898-68622128

\section{Abstract}

Background: It has been reported that the NF-KB and MMP-9 signaling pathways were involved in the pathogenesis of osteoarthritis (OA), while the treatment of OA by YSYO1 could inhibit the proteasome activity. Therefore, we aimed to study the therapeutic effect of YSY01A treatment on OA via interfering with expression of NF-KB and MMP-9. Methods: Western blot analysis and immunohistochemistry (IHC) assays were used to measure the expression of NF-KB and MMP-9 in animal models established via SH treatment or cellular models established via sodium nitroprusside (SNP) treatment. MTT assay and flow cytometry analysis were performed to observe the effect of YSY01 treatment on cell viability and apoptosis. Results: The decreased expression of NF-KB and MMP-9 was observed in OA rabbits and cells treated by YSYO1A, thus indicating the inhibitory effect of YSY01A on NF-KB and MMP-9 expression. And YSY01A exhibited a positive therapeutic effect on OA both in vivo and in vitro by inhibiting the expression of NF-KB and MMP-9. Meanwhile, YSY01A treatment could increase cell viability to a certain degree and decrease the apoptosis index, which suggested the application of YSY01A in OA therapy. Conclusions: The levels of NF-KB and MMP-9 which were associated with aggravated 
apoptosis were up-regulated in OA models in vivo and in vitro. YSY01A treatment could down-regulate the expression of NF-KB and MMP-9 and inhibit cell apoptosis, thus reducing the severity of $O A$.

Running title: YSY01A treatment of OA via modulating NF-kB/MMP-9.

Key words: Osteoarthritis; YSY01A; NF-kB; MMP-9; Apoptosis

\section{Introduction}

As a frequently diagnosed joint disease, osteoarthritis (OA) affects nearly $15 \%$ of global population and can lead to disability or severe pain [1, 2]. Although traditional therapies can alleviate the symptoms of OA to a certain degree, the progression of OA cannot be absolutely prevented in all cases. Thus, novel approaches are necessary for the treatment of OA to relieve the pain. The degeneration of OA cartilage is prominent with chondrocyte proliferation, which lead to cell death accordingly. And the apoptotic bodies derived from chondrocytes can also lead to the aberrant calcification in the cartilage matrix due to their capability to precipitate calcium [3]. Therefore, chondrocyte clusters can induce calcification in the cartilage matrix a well as the growth and proliferation of chondrocytes, and the activity of chondrocyte clusters is associated with the death of chondrocytes and the generation of apoptotic bodies [4, 5]. Moreover, apoptosis is also an important index for the treatment of various diseases via different durgs [6-10]. For example, etanercept could protect ovarian reserve against ischemia-reperfusion injury (IRI) via regulating apoptosis [7], eriodictyol exerted its anticancer activity through induction of mitochondrial apoptosis in the treatment of lung cancer [8], and salvianolic acid A had a synergistically protective effect on hepatic IRI via hepatocellular apoptosis. Moreover, 5methoxypsoralen could exert apoptotic effects in U-87MG human glioma cells [9] and glucose fluctuation could also increase mesangial cell apoptosis [10].

It was shown that up-regulated nuclear factor-kB1 (NF- KB) expression is induced via the inhibition of miR-9 expression, thus leading to increased cell proliferation during knee OA. The up-regulated NF-KB expression might be explained by the differences of cell types and intercellular environment. In fact, miR-9 is lowly expressed in the knee OA 
chondrocytes and its low expression is associated with increased chondrocyte apoptosis [11]. Besides, miR-9 can negatively regulate NF-KB expression, thereby the downregulated miR-9 expression will increase NF-kB expression and suppress cell proliferation $[12,13]$.Furthermore, NF-KB can simultaneously control a wide range of genes with different functions (e.g., genes with anti-apoptotic and anti-proliferative effects), and NFkB may facilitate higher expression of anti-apoptotic genes in chondrocytes. Nevertheless, the expressional imbalance of genes in different types of cells should be further explored to verify the above hypothesis. The elevation of MMP expression can lead to the destruction of cartilage during OA. So far, as many as seven MMPs have been identified in articular cartilage, such as MMP-9 (also named gelatinase B) [14]. MMP-9 expression is apparently increased in the articular cartilage of OA to aggravate collagen degeneration $[2,15]$. The expression of MMP-9 gene is regulated by upstream regulatory factors such as AP-1 and NF-KB [16]. In fact, some of these factors were involved in regulating the functions of a wide range of agents. Subsequently, the inhibition of c-Jun, c-Fos and NFKB activity can potentially inhibit tumor metastasis $[17,18]$. It is also likely that the suppressive role of HLE in the invasion and metastasis of LNCaP cells is mediated by NF$\mathrm{KB}$ and MMP-9 inactivation.

YSY01A (N-(2-pyrazinecarbonyl) -L-leucine-L-(2-naphthyl)- alanine-L- leucine boronic acid) is a newly developed proteasome blocker with anti-tumor effects, which is actively participating in the anti-cancer activity in vitro [19]. YSYO1A has been found to inhibit proteasome activity and alter the expression of multiple genes, thus modulated a wide range of signaling pathways implicated in autophagy, cell apoptosis, cell cycle, and cell proliferation [20-23]. Specifically, Zhang et al. reported that the anti-tumor effect of YSY01A is completed by arresting the cell cycle at the G2/M stage [20]. Xue et al. indicated YSY01A as a novel therapeutic agent for breast cancer treatment since it exhibited a potential therapeutic effects for breast cancer MCF-7 by inducing G2 phase arrest via ER $\alpha$ and PI3K/Akt pathways [21]. Huang et al. also demonstrated that YSY01A could suppress the survival and migration of A549 non-small cell lung cancer cells by abrogating constitutively active STAT3 signaling cascade [22] or the survival of cisplatin-resistant 
ovarian cancer cells by inducing apoptosis [23].The signaling pathways of NF-kB and MMP-9 have been reported to participate in the pathogenesis of OA [24]. In this study, we examined the effect of YSY01A on the expression of NF-kB and MMP-9, so as to identify the therapeutic effect of YSYO1A in the treatment of OA.

\section{Materials and Methods}

\section{OA model establishment}

To examine the therapeutic effect of YSY01A in the treatment of OA, we established a rabbit model of OA by treating rabbits with $1 \%$ SH in DMEM (Gibco, Thermo Fisher Scientific, Waltham, MA). Subsequently, the rabbits were divided into a sham-operated group, an OA group, and an OA + YSY01A treatment group ( $N=12$ in each treatment group and 3 samples collected from each animal). The animals were fed with regular water and food under room temperature. In the OA group, each rabbit was given an i.p. injection of $3.5 \mathrm{~mL} / \mathrm{kg}$ chloral hydrate (Sigma-Aldrich, St. Louis, MO) for anesthesia and skin of left knee was shaved before a $2 \mathrm{~cm}$ longitudinal incision was cut from above the medial knee joint, over the medial collateral ligament, and to the posterior cruciate ligament, followed by the removal of the medial meniscus. Subsequently, the drawer test was performed to confirm it and the normal saline was used to rinse the joint cavity before the skin and capsule were sutured without fixation. The rabbits in the OA + YSY01A group were administered once/day injections of $1 \%$ YSY01A $(0.2 \mathrm{mg} / \mathrm{kg})$ into the intra-articular cavity for 5 consecutive weeks. This animal experiment was approved by the ethics committee at Hainan General Hospital (Approval number: NDYS003201748). All animal experiments in this study were done following the guidelines published by the National Institutes of Health on the Management and Use of Laboratory Animals.

\section{OA cell model}

To further validate the positive therapeutic effect of YSY01A treatment on OA, rabbit osteoblast cells and HFOB1.19 cells were treated with SNP to establish a cellular model of OA. The cells were cultured at $37{ }^{\circ} \mathrm{C}$ and $5 \% \mathrm{CO}_{2}$ in a $10 \%$ fetal bovine serum-containing 
DMEM medium supplemented with antibiotics. The cells were divided into a control group, an SNP group, and an SNP + YSYO1A treatment group. The cells in the SNP group and the SNP + YSY01A group were treated for $24 \mathrm{~h}$ with $2 \mathrm{mM}$ SNP. The cells in the SNP + YSY01A group were also treated with 0, $25 \mathrm{nM}$, or $50 \mathrm{nM}$ YSY01A at the same time of SNP treatment.

\section{RNA isolation and real-time PCR}

The tissue and cell samples were homogenized using a saline solution, and the total RNA was extracted with a Trizol RNA Extraction Kit (Invitrogen, Carlsbad, CA). The total RNA was converted into cDNA using an RT PrimeScript Kit (Invitrogen, Carlsbad, CA) following the manufacturer's instructions. The volume of the RT-PCR system was $10 \mu \mathrm{l}$ and the reaction condition was $37^{\circ} \mathrm{C}$. The $\mathrm{RT}$ procedures included 3 cycles of reverse transcription (15 min each) and a $5 \mathrm{~s}$ denaturation cycle at $85^{\circ} \mathrm{C}$. The mRNA expression of NF-kB and MMP-9 was detected by real-time RT-PCR using a Premix Ex Taqll SYBR kit (ABI, Forster City, CA). The reaction procedures included $15 \mathrm{~min}$ pre-denaturation at $95^{\circ} \mathrm{C}$, followed by 40 cycles of $15 \mathrm{~s}$ denaturation at $95^{\circ} \mathrm{C}$, and $60 \mathrm{~s}$ annealing and extension at $60^{\circ} \mathrm{C}$. The reaction system included $2 \mu \mathrm{l}$ of upstream PCR primer, $2 \mu \mathrm{l}$ of downstream primer, $4 \mu \mathrm{l}$ of DNA template, $1 \mu$ l of ROX Reference Dye (50x), $25 \mu$ l of SYBR Premix Ex Taqll, and $16 \mu \mathrm{l}$ of $\mathrm{dH}_{2} \mathrm{O}$. The expression of GAPDH was used as the internal reference. The expression of NF-KB and MMP-9 mRNA was calculated using the $2^{-\Delta \Delta \mathrm{Ct}}$ method, where $\mathrm{Ct}$ is the cycle threshold value.

\section{Cell proliferation assay}

Isolated rabbit osteoblast cells and HFOB1.19 cells were seeded into 96-well plates at a concentration of $5 \times 10^{3}$ cells per well. The cells in different treatment groups were treated according. Subsequently, each well received $200 \mu$ l of DMEM media containing $10 \%$ FBS and cultured at $37^{\circ} \mathrm{C}$ with $5 \% \mathrm{CO}_{2}$ for $12,24,48 \mathrm{~h}, 72 \mathrm{~h}$ and $96 \mathrm{~h}$. In each well, $20 \mu \mathrm{l}$ of MTT (diluted to $5 \mathrm{mg} / \mathrm{ml}$ with PBS) were added and incubated for $4 \mathrm{~h}$. After discarding the supernatant, $150 \mu \mathrm{l}$ of dimethyl sulfoxide (DMSO) were added into each well. The plates were gently shaken on an oscillator for $10 \mathrm{~min}$ and were measured in a microplate reader. 
The optical densities (OD) at $490 \mathrm{~nm}$ were measured on a NanoDrop ND-1000 spectrophotometer (NanoDrop Technologies, Wilmington, DE). Using the time as the $\mathrm{x}$ axis and the OD values as the y-axis, a viability curve was plotted to analyze the cell viability in different treatment groups.

\section{Western blot analysis}

A total protein extraction rapid immunofilter paper assay (RIPA) reagent kit (Beyotime Biotechnology, Shanghai, China) was used to extract the total protein from tissue and cell samples. A bicinchoninic acid (BCA) assay kit was used to determine the protein concentration. After protein separation by polyacrylamide gel electrophoresis, the proteins were transferred to a nitrocellulose (NC) membrane by a wet transfer method. The membrane was then blocked with $5 \%$ bovine serum albumin (BSA) for $1 \mathrm{~h}$ at room temperature and incubated with diluted primary antibodies against NF-kB (1:1000, ab16502, Abcam, Cambridge, MA), MMP-9 (1:1000, ab38898, Abcam, Cambridge, MA) and GAPDH (1:1000, ab9485, Abcam, Cambridge, MA) at $4^{\circ} \mathrm{C}$ overnight. The membrane was subsequently washed with PBS and further incubated with HRP-labeled IgG secondary antibodies (1:1500, ab6721 Sigma-Aldrich, Merck KGaA, Darmstadt, Germany) at room temperature for $1 \mathrm{~h}$. The membrane was developed in an electrochemiluminescence (ECL) solution (Pierce, Rockford, IL) and the images of the gels were acquired in a dark room. A Western blot semi quantitative method was then used to measure the expression of target proteins using GAPDH as the internal control. The gray values of the protein bands were analyzed by Image J software.

\section{Apoptosis analysis}

Treated rabbit osteoblast cells and HFOB1.19 cells were harvested by centrifugation at $1000 \mathrm{rpm}$ and $4^{\circ} \mathrm{C}$ for $5 \mathrm{~min}$. The cell pellets were washed twice with PBS and fixed at $4^{\circ} \mathrm{C}$ overnight in cold $70 \%$ ethanol. Subsequently, the samples were incubated at $37^{\circ} \mathrm{C}$ for 5 min with RNase enzyme, and stained in the dark for 30 min with PI and FITC reagents using an Annexin-V-FITC/PI apoptosis detection kit (Thermo Fisher Scientific, Waltham, 
MA). The apoptosis profile of the samples was measured on a FACSCalibur flow cytometer (BD Biosciences, Franklin Lakes, NJ).

\section{Immunohistochemistry}

Histochemical immunostaining was done using the streptavidin-peroxidase three-step method. All specimens were fixed in $4 \%$ formalin and embedded in paraffin. The specimens were then sliced continuously to a slice thickness of $2 \mu \mathrm{m}$. Antigens on the tissue sections were repaired by boiling in a microwave, and the slides were subsequently immersed in $3 \% \mathrm{H}_{2} \mathrm{O}_{2}$ deionized water for $10 \mathrm{~min}$ at room temperature to block endogenous oxidase activity. Subsequently, non-immune sera were added on the slides dropwisely to remove impurities in the antigen. The slides were then incubated at room temperature for $30 \mathrm{~min}$ and washed three times with PBS. Subsequently, the primary antibodies against NF-kB and MMP-9 (Abcam, Cambridge, MA) were added on the slides dropwisely and the slides were incubated overnight at $4^{\circ} \mathrm{C}$. After PBS washing, secondary antibodies were added on the slides dropwisely and the slides were incubated at room temperature for $10 \mathrm{~min}$. The reaction was terminated by adding an anti-biotin-labeled peroxidase solution, and the slides were colorized with DAB. After re-staining with hematoxylin, the nuclei were revealed using PBS. Finally, the slides were dehydrated with anhydrous ethanol and dried before being mounted with neutral gum. The results of immunostaining were observed under a 400x microscope.

\section{Statistical analysis}

All data were analyzed using the SPSS 19.0 statistical software. All measurement data were expressed by mean \pm standard deviation. The comparison between two groups was carried out using t-tests, while the comparison among multiple groups was carried out using one-way ANOWA. A probability value of $P<0.05$ was considered statistically significant.

\section{Results}


To examine the therapeutic effect of YSY01A in the treatment of OA, we established an OA rabbit model, which was divided into a sham-operated group, an OA group, and an $\mathrm{OA}+$ YSY01A treatment group. Subsequently, the expression levels of NF-KB and MMP-9 were measured. As shown in Fig.1A, the protein bands of NF-KB and MMP-9 were much denser in the OA group compared with those in the sham-operated group or OA + YSY01A treatment group, and the relative density of NF-KB protein (Fig.1B) and MMP-9 protein (Fig.1C) was clearly elevated in the OA group compared with that in the sham-operated group. However, YSY01A treatment reduced the increased expression of NF-KB and MMP9 in OA rabbits to the normal level. Furthermore, IHC assay was performed to validate the inhibitory effect of YSY01A on the protein expression of NF-KB (Fig.2) and MMP-9 (Fig.3). Accordingly, NF-KB (Fig.2) and MMP-9 (Fig.3) showed relatively higher expression in the OA rabbits, while YSY01A treatment reduced the NF-KB and MMP-9 expression to a level similar as that in the sham-operated group, indicating that the YSYO1A treatment could reduce the expression of NF-KB and MMP-9, thus exhibiting a positive therapeutic effect on OA.

\section{YSY01A treatment inhibited NF-KB and MMP-9 expression in vitro}

To further validate the positive therapeutic effect of YSY01A treatment in OA, rabbit osteoblast cells and HFOB1.19 cells were treated by SNP to establish a cellular model of $\mathrm{OA}$, which was then divided into a control group, an SNP group, and an SNP + YSY01A treatment group. Accordingly, the protein expression levels of NF-KB (Fig.4A and 4B) and MMP-9 (Fig.4A and 4C) in the OA model of rabbit osteoblast cells were significantly higher than those in the control group, which were subsequently down-regulated by the YSY01A treatment. Also, similarly results were obtained when the above observations were repeated in HFOB1.19 cells (Fig.5), validating the inhibitory effect of YSY01A treatment on NF-KB and MMP-9 expression in vitro.

\section{YSY01A treatment could alleviate cell apoptosis and increase cell viability}

Since SNP treatment could induce cell apoptosis in vitro, we observed cell viability of rabbit osteoblast cells and HFOB1.19 cells up to 96h via MTT assays. As demonstrated in 
Fig.6A, the control group of rabbit osteoblast cells showed the highest cell viability, whereas the SNP group of rabbit osteoblast cells showed the lowest cell viability. Significantly, the YSY01A treatment group could increase the cell viability to a certain extent, although the cell viability in the SNP + YSY01A treatment group was still lower than that in the control group. In addition, as shown in Fig.6B, the apoptosis index (\%) evaluated in rabbit osteoblast cells via flow cytometry showed a twofold increase of the apoptosis index in the SNP group compared with that in the control group, whereas YSY01A treatment decreased the value of apoptosis index to a certain extent. And similar results were also obtained when the above observations were repeated in HFOB1.19 cells (Fig.7). Therefore, this study demonstrated a molecular mechanism underlying the therapeutic role of YSY01A in the treatment of OA. In summary, YSY01A treatment can alleviate the severity of OA by down-regulating the increased expression of NF-KB and MMP-9 in OA, and YSY01A treatment can also promote cell proliferation while obstruct cell apoptosis in OA.

\section{Discussion}

It was found that chondrocyte apoptosis becomes more apparent in the articular cartilage of OA. In fact, the percentage of apoptotic cells is the highest in the middle and superficial zones of OA cartilage. In addition, the number of apoptotic chondrocytes is correlated to donor age.

In this study, we used an OA rabbit model to examine the therapeutic effect of YSYO1A in the treatment of OA. The decreased expression of NF-KB and MMP-9 was observed upon YSY01A treatment both in vitro and in vivo. Therefore, YSY01A treatment was demonstrated to exhibit its therapeutic effect in OA treatment by inhibiting the expression of NF-KB and MMP-9.

NF-kB1 controls the expression of many genes implicated in apoptosis, cell proliferation, angiogenesis, cell differentiation, cell adhesion, and immune response [25]. NF-kB is also necessary the full activation of MMP-9 via TNF-a [26]. Furthermore, it was found that miR9 mimics suppressed the NF-kB1 protein expression in knee OA chondrocytes, while the 
downregulation of miR-9 could trigger the increase in NF-kB1 expression at both translation and transcription levels in the chondrocytes. Hence, it has been suspected that the expression of NF-kB1 at both mRNA and protein levels is modulated by miR-9. Besides, the interaction between miR-9 and NF-kB1 is shown to suppress apoptosis during chondrogenesis [6]. The competition between NF-kB and p65 subunit for the binding with MMP-9 can suppress the expression of MMP-9, thus leading to the transcriptional activation of NF-kB [27, 28]. Moreover, NF-kB activity can control TNF-a expression [29].

As a superfamily of zinc-dependent proteolytic enzyme, MMP is implicated in the degradation of their target proteins by cleaving the internal peptide bonds [30]. MMP-9 is a highly complex member in the MMP family. The proteolytic activity of MMP-9 against type-IV collagen is increased in multiple types of human cancers, such as esophageal, breast and gastric cancer [31]. MMP-9 also plays a critical role in craniofacial development and skeletogenesis [32-38]. Moreover, MMP-9 controls the apoptosis of chondrocytes and the progression of OA $[32,39]$. In MMP-9 deficient mice, the degree of ossification, vascularization, and chondrocyte apoptosis is significantly reduced, thus promoting the plate growth [39]. MMP-9 also plays an important role in inducing the apoptosis of chondrocytes in the bones $[39,40]$. The simultaneous expression of MMP-9 in endochondral skeletons and cranial base showed that MMP-9 is involved in mediating the apoptosis of chondrocytes and the angiogenesis in endochondral skeletons. It was shown that the high levels of MMP-9, MMP-2, and MMP-1 in OA may promote its progression. OA can lead to severe pain and stiffness in elder people. In addition, OA is characterized by the gradual articular cartilage destruction in the synovial joints [41]. The expression of MMP-9, MMP-2, and MMP-1 is very high in OA and greatly damages the extracellular matrix, thus inducing tumor metastasis and invasion via tissue remodeling [42]. It was found that the protein expression of MMP-9, MMP-2, and MMP-1 was significantly higher in OA patients compared with that in normal people. In this study, the cell viability and cell apoptosis index were examined. The results showed that YSY01A treatment could restore the viability of OA cells and decrease their apoptosis to a certain extent. Therefore, a molecular mechanism underlying the therapeutic effect of YSY01A in OA therapy was 
revealed in this study, which suggested that YSY01A played a beneficial role in the treatment of OA by promoting cell proliferation and by inhibiting cell apoptosis. However, although we examined the therapeutic effect of YSY01A in the treatment of OA in a OA rabbit model and a cellular model of OA in this study, a continued testing on human participants should be conducted to validate the clinical value of our finding, which is the limitation of our study.

\section{Conclusion}

Taken together, the findings of this study demonstrated that YSY01A treatment could exert a therapeutic effect on OA by down-regulating the up-regulated expression of NFKB and MMP-9 in OA. In addition, YSY01A treatment could also promote cell proliferation and inhibit cell apoptosis.

\section{Conflict of interest}

None

\section{Figure legends}

\section{Fig.1}

NF-KB and MMP-9 were highly expressed in OA rabbits

A. The density of NF-KB and MMP-9 protein bands was higher in the OA group compared with that in the sham-operated group or the OA + YSY01A treatment group;

B. Relative density of NF-KB was increased in the OA group;

C. Relative density of MMP-9 was increased in the OA group.

\section{Fig.2}

IHC assay showed higher expression of NF-KB in the OA group compared with that in the sham-operated group, and YSYO1A treatment could reduce the expression of NF-KB in OA rabbits.

\section{Fig.3}


IHC assay showed higher expression of MMP-9 in the OA group compared with that in the sham-operated group, and YSY01A treatment could reduce the expression of MMP-9 in OA rabbits.

\section{Fig.4}

YSY01A treatment showed an inhibitory effect on NF-KB and MMP-9 expression in an OA model of rabbit osteoblast cells.

A. The density of NF-KB and MMP-9 protein bands was higher in the SNP group compared with that in the control group or SNP + YSY01A treatment group;

B. Relative density of NF-KB was increased in the SNP group;

C. Relative density of MMP-9 was increased in the SNP group.

\section{Fig.5}

YSY01A treatment showed an inhibitory effect on NF-KB and MMP-9 expression in an OA model of HFOB1.19 cells.

A. The density of NF-KB and MMP-9 protein bands was higher in the SNP group compared with that in the control group or SNP + YSYO1A treatment group;

B. Relative density of NF-KB was increased in the SNP group;

C. Relative density of MMP-9 was increased in the SNP group.

\section{Fig.6}

YSY01A treatment could partly alleviate cell apoptosis and restore the viability of rabbit osteoblast cells cells.

A. A step-wise decrease of cell viability was observed in the order of control group, SPN + YSY01A treatment group, and SNP group;

B. Cell apoptosis index (\%) of SNP group showed a twofold increase compared with that in the control group, whereas YSY01A treatment decreased the cell apoptosis index to a certain degree.

Fig.7 
YSY01A treatment could partly alleviate cell apoptosis and restore the viability of HFOB1.19 cells.

A. A step-wise decrease of cell viability was observed in the order of control group, SPN + YSY01A treatment group, and SNP group;

B. Cell apoptosis index (\%) of SNP group showed a twofold increase compared with that in the control group, whereas YSY01A treatment decreased the cell apoptosis index to a certain degree.

\section{References}

1 Huskisson EC: Glucosamine and chondroitin for osteoarthritis. J Int Med Res 2008;36:1161-1179.

2 Soder S, Roach HI, Oehler S, Bau B, Haag J, Aigner T: MMP-9/gelatinase B is a gene product of human adult articular chondrocytes and increased in osteoarthritic cartilage. Clin Exp Rheumatol 2006;24:302-304.

3 Hashimoto S, Ochs RL, Rosen F, Quach J, McCabe G, Solan J, Seegmiller JE, Terkeltaub R, Lotz M: Chondrocyte-derived apoptotic bodies and calcification of articular cartilage. Proc Natl Acad Sci U S A 1998;95:3094-3099.

4 Gibson GJ, Kohler WJ, Schaffler MB: Chondrocyte apoptosis in endochondral ossification of chick sterna. Dev Dyn 1995;203:468-476.

5 Roach HI, Erenpreisa J, Aigner T: Osteogenic differentiation of hypertrophic chondrocytes involves asymmetric cell divisions and apoptosis. J Cell Biol 1995;131:483-494.

6 Yang L, Jiang L, Jiang D, Liu B, Jin S: The protective effects of salvianolic acid A against hepatic ischemia-reperfusion injury via inhibiting expression of toll-like receptor 4 in rats. Arch Med Sci. 2019 Oct; 15(6): 1599-1607.

7 Eken MK, Ersoy GS, Kaygusuz El, Devranoğlu B, Takır M, Çilingir OT, Çevik O: Etanercept protects ovarian reserve against ischemia/reperfusion injury in a rat model. Arch Med Sci 2019; 15(4): 1104-1112.

8 Zhang $\mathrm{Y}$, Zhang $\mathrm{R}, \mathrm{Ni} \mathrm{H}$ : Eriodictyol exerts potent anticancer activity against A549 human lung cancer cell line by inducing mitochondrial-mediated apoptosis, G2/M 
cell cycle arrest and inhibition of m-TOR/PI3K/Akt signalling pathway. Arch Med Sci 2020; 16(2): 446-452.

9 Guo H, He Y, Bu C, Peng Z: Antitumor and apoptotic effects of 5-methoxypsoralen in U87MG human glioma cells and its effect on cell cycle, autophagy and PI3K/Akt signaling pathway. Arch Med Sci 2019; 15(6): 1530-1538.

10 Ying C, Wang S, Lu Y, Chen L, Mao Y, Ling H, Cheng X, Zhou X: A Glucose fluctuation increased mesangial cell apoptosis related to AKT signal pathway. Arch Med Sci 2019; 15(3): 730-737.

11 Song J, Kim D, Chun CH, Jin EJ: MicroRNA-9 regulates survival of chondroblasts and cartilage integrity by targeting protogenin. Cell Commun Signal 2013;11:66.

12 Liu N, Sun Q, Chen J, Li J, Zeng Y, Zhai S, Li P, Wang B, Wang X: MicroRNA-9 suppresses uveal melanoma cell migration and invasion through the NF-kappaB1 pathway. Oncol Rep 2012;28:961-968.

13 Imagawa K, de Andres MC, Hashimoto K, Pitt D, Itoi E, Goldring MB, Roach HI, Oreffo RO: The epigenetic effect of glucosamine and a nuclear factor-kappa B (NFkB) inhibitor on primary human chondrocytes--implications for osteoarthritis. Biochem Biophys Res Commun 2011;405:362-367.

14 Rose BJ, Kooyman DL: A Tale of Two Joints: The Role of Matrix Metalloproteases in Cartilage Biology. Dis Markers 2016;2016:4895050.

15 Freemont AJ, Hampson V, Tilman R, Goupille P, Taiwo Y, Hoyland JA: Gene expression of matrix metalloproteinases 1, 3, and 9 by chondrocytes in osteoarthritic human knee articular cartilage is zone and grade specific. Ann Rheum Dis 1997;56:542-549.

16 Westermarck J, Kahari VM: Regulation of matrix metalloproteinase expression in tumor invasion. FASEB J 1999;13:781-792.

17 Lee YC, Lin HH, Hsu CH, Wang CJ, Chiang TA, Chen JH: Inhibitory effects of andrographolide on migration and invasion in human non-small cell lung cancer A549 cells via down-regulation of PI3K/Akt signaling pathway. Eur J Pharmacol 2010;632:23-32. 
18 Philip S, Kundu GC: Osteopontin induces nuclear factor kappa B-mediated promatrix metalloproteinase-2 activation through I kappa B alpha /IKK signaling pathways, and curcumin (diferulolylmethane) down-regulates these pathways. J Biol Chem 2003;278:14487-14497.

19 Zhu Y, Yao S, Xu B, Ge Z, Cui J, Cheng T, Li R: Design, synthesis and biological evaluation of tripeptide boronic acid proteasome inhibitors. Bioorg Med Chem 2009;17:6851-6861.

20 Zhang M, Yuan X, Xu B, Guo W, Ran FX, Li RT, Cui JR: Anticancer Effect of a Novel Proteasome Inhibitor, YSY01A, via G2/M Arrest in PC-3M Cells in vitro and in vivo. J Cancer 2015;6:701-708.

21 Xue B, Huang W, Yuan X, Xu B, Lou Y, Zhou Q, Ran F, Ge Z, Li R, Cui J: YSY01A, a Novel Proteasome Inhibitor, Induces Cell Cycle Arrest on G2 Phase in MCF-7 Cells via ERalpha and PI3K/Akt Pathways. J Cancer 2015;6:319-326.

22 Huang W, Yuan X, Sun T, Fan S, Wang J, Zhou Q, Guo W, Ran F, Ge Z, Yang H, Li R, Cui J: Proteasome Inhibitor YSY01A Abrogates Constitutive STAT3 Signaling via Down-regulation of Gp130 and JAK2 in Human A549 Lung Cancer Cells. Front Pharmacol 2017;8:476.

23 Huang W, Zhou Q, Yuan X, Ge ZM, Ran FX, Yang HY, Qiang GL, Li RT, Cui JR: Proteasome Inhibitor YSY01A Enhances Cisplatin Cytotoxicity in CisplatinResistant Human Ovarian Cancer Cells. J Cancer 2016;7:1133-1141.

24 Xu X, Lv H, Li X, Su H, Zhang X, Yang J: Danshen attenuates osteoarthritis-related cartilage degeneration through inhibition of NF-kappaB signaling pathway in vivo and in vitro. Biochem Cell Biol 2017;95:644-651.

25 Gu R, Liu N, Luo S, Huang W, Zha Z, Yang J: MicroRNA-9 regulates the development of knee osteoarthritis through the NF-kappaB1 pathway in chondrocytes. Medicine (Baltimore) 2016;95:e4315.

26 Xue H, Sun K, Xie W, Hu G, Kong H, Wang Q, Wang H: Etanercept attenuates shortterm cigarette-smoke-exposure-induced pulmonary arterial remodelling in rats by 
suppressing the activation of TNF-a/NF-kB signal and the activities of MMP-2 and MMP-9. Pulm Pharmacol Ther 2012;25:208-215.

27 Sanceau J, Boyd DD, Seiki M, Bauvois B: Interferons inhibit tumor necrosis factoralpha-mediated matrix metalloproteinase-9 activation via interferon regulatory factor-1 binding competition with NF-kappa B. J Biol Chem 2002;277:35766-35775.

28 Choo EJ, Rhee YH, Jeong SJ, Lee HJ, Kim HS, Ko HS, Kim JH, Kwon TR, Jung JH, Lee EO, Kim DK, Chen CY, Kim SH: Anethole exerts antimetatstaic activity via inhibition of matrix metalloproteinase $2 / 9$ and AKT/mitogen-activated kinase/nuclear factor kappa B signaling pathways. Biol Pharm Bull 2011;34:41-46.

29 Rodriguez M, Cabal-Hierro L, Carcedo MT, Iglesias JM, Artime N, Darnay BG, Lazo PS: NF-kappaB signal triggering and termination by tumor necrosis factor receptor 2. J Biol Chem 2011;286:22814-22824.

30 Bourboulia D, Stetler-Stevenson WG: Matrix metalloproteinases (MMPs) and tissue inhibitors of metalloproteinases (TIMPs): Positive and negative regulators in tumor cell adhesion. Semin Cancer Biol 2010;20:161-168.

31 De Groef L, Van Hove I, Dekeyster E, Stalmans I, Moons L: MMPs in the neuroretina and optic nerve: modulators of glaucoma pathogenesis and repair? Invest Ophthalmol Vis Sci 2014;55:1953-1964.

32 Vu TH, Werb Z: Matrix metalloproteinases: effectors of development and normal physiology. Genes Dev 2000;14:2123-2133.

33 Blagden CS, Hughes SM: Extrinsic influences on limb muscle organisation. Cell Tissue Res 1999;296:141-150.

34 Mukhopadhyay M, Shtrom S, Rodriguez-Esteban C, Chen L, Tsukui T, Gomer L, Dorward DW, Glinka A, Grinberg A, Huang SP, Niehrs C, Izpisua Belmonte JC, Westphal H: Dickkopf1 is required for embryonic head induction and limb morphogenesis in the mouse. Dev Cell 2001;1:423-434.

35 Day TF, Guo X, Garrett-Beal L, Yang Y: Wnt/beta-catenin signaling in mesenchymal progenitors controls osteoblast and chondrocyte differentiation during vertebrate skeletogenesis. Dev Cell 2005;8:739-750. 
36 Hill TP, Spater D, Taketo MM, Birchmeier W, Hartmann C: Canonical Wnt/betacatenin signaling prevents osteoblasts from differentiating into chondrocytes. Dev Cell 2005;8:727-738.

37 Hu H, Hilton MJ, Tu X, Yu K, Ornitz DM, Long F: Sequential roles of Hedgehog and Wnt signaling in osteoblast development. Development 2005;132:49-60.

38 Li X, Liu P, Liu W, Maye P, Zhang J, Zhang Y, Hurley M, Guo C, Boskey A, Sun L, Harris SE, Rowe DW, Ke HZ, Wu D: Dkk2 has a role in terminal osteoblast differentiation and mineralized matrix formation. Nat Genet 2005;37:945-952.

39 Vu TH, Shipley JM, Bergers G, Berger JE, Helms JA, Hanahan D, Shapiro SD, Senior RM, Werb Z: MMP-9/gelatinase $B$ is a key regulator of growth plate angiogenesis and apoptosis of hypertrophic chondrocytes. Cell 1998;93:411-422.

40 Engsig MT, Chen QJ, Vu TH, Pedersen AC, Therkidsen B, Lund LR, Henriksen K, Lenhard T, Foged NT, Werb Z, Delaisse JM: Matrix metalloproteinase 9 and vascular endothelial growth factor are essential for osteoclast recruitment into developing long bones. J Cell Biol 2000;151:879-889.

41 Chen YJ, Tsai KS, Chan DC, Lan KC, Chen CF, Yang RS, Liu SH: Honokiol, a low molecular weight natural product, prevents inflammatory response and cartilage matrix degradation in human osteoarthritis chondrocytes. J Orthop Res 2014;32:573-580.

42 Roy R, Yang J, Moses MA: Matrix metalloproteinases as novel biomarkers and potential therapeutic targets in human cancer. J Clin Oncol 2009;27:5287-5297. 
A

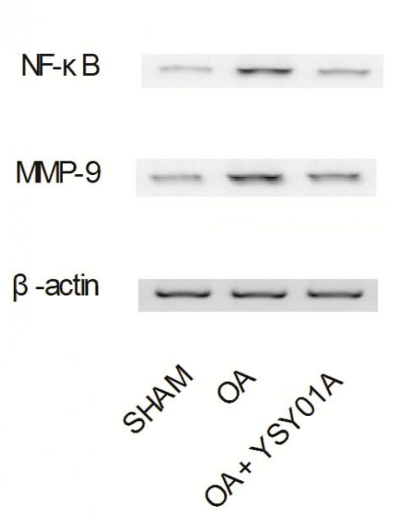

B

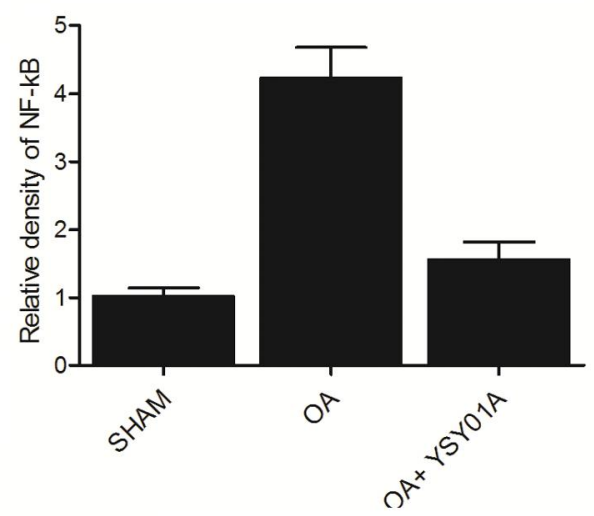

C

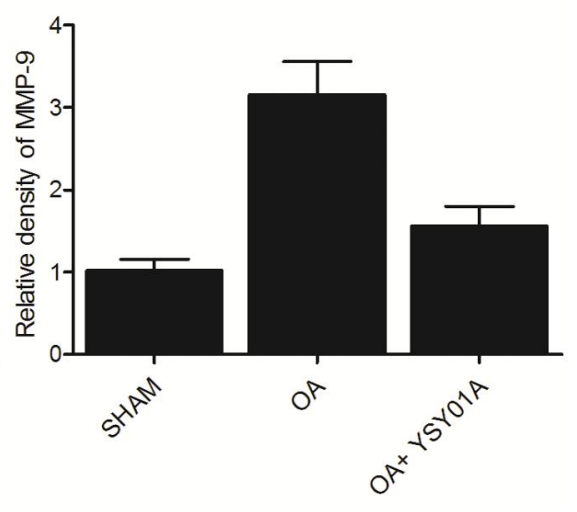


OA

OA+ YSY01A

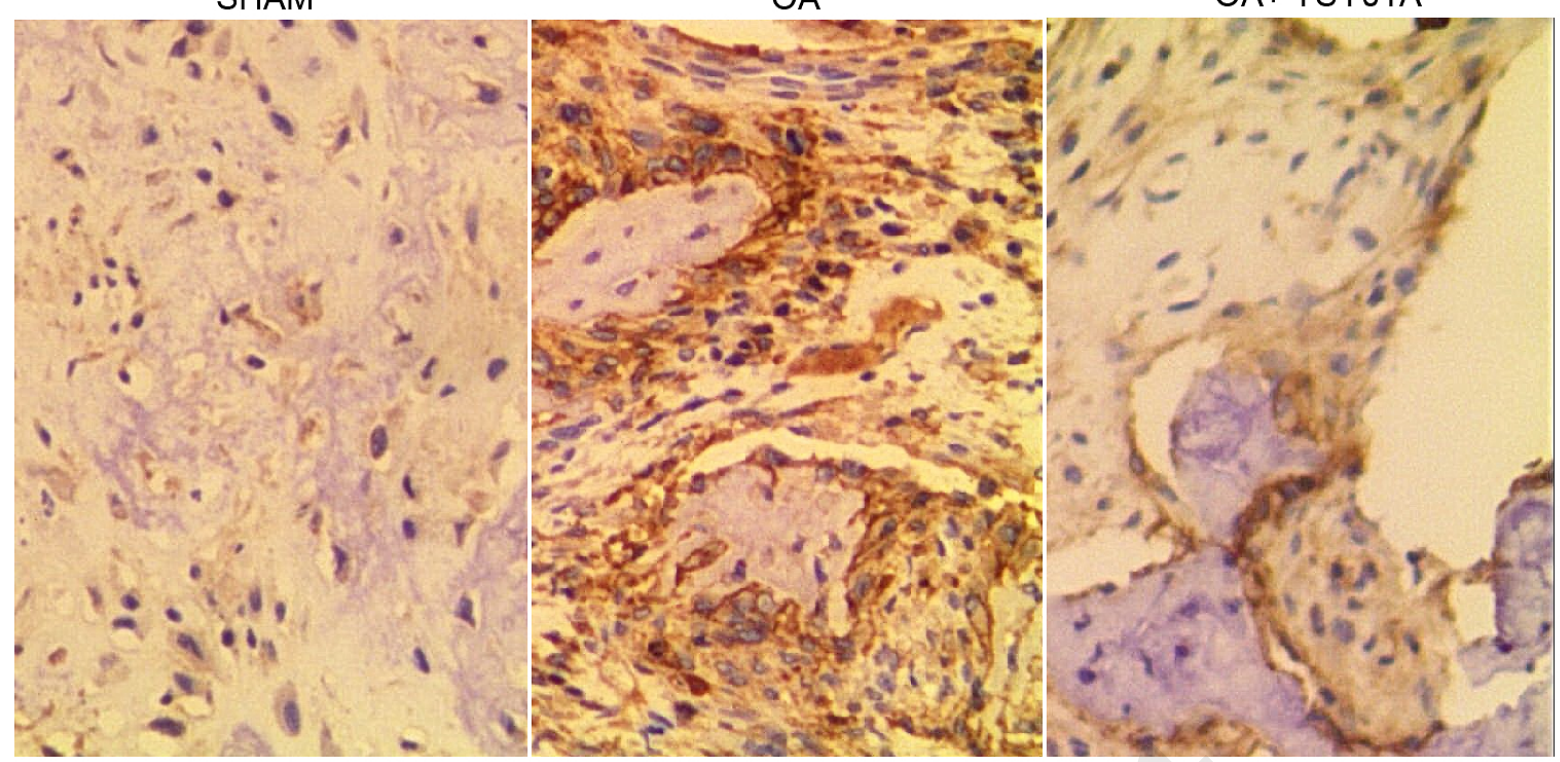



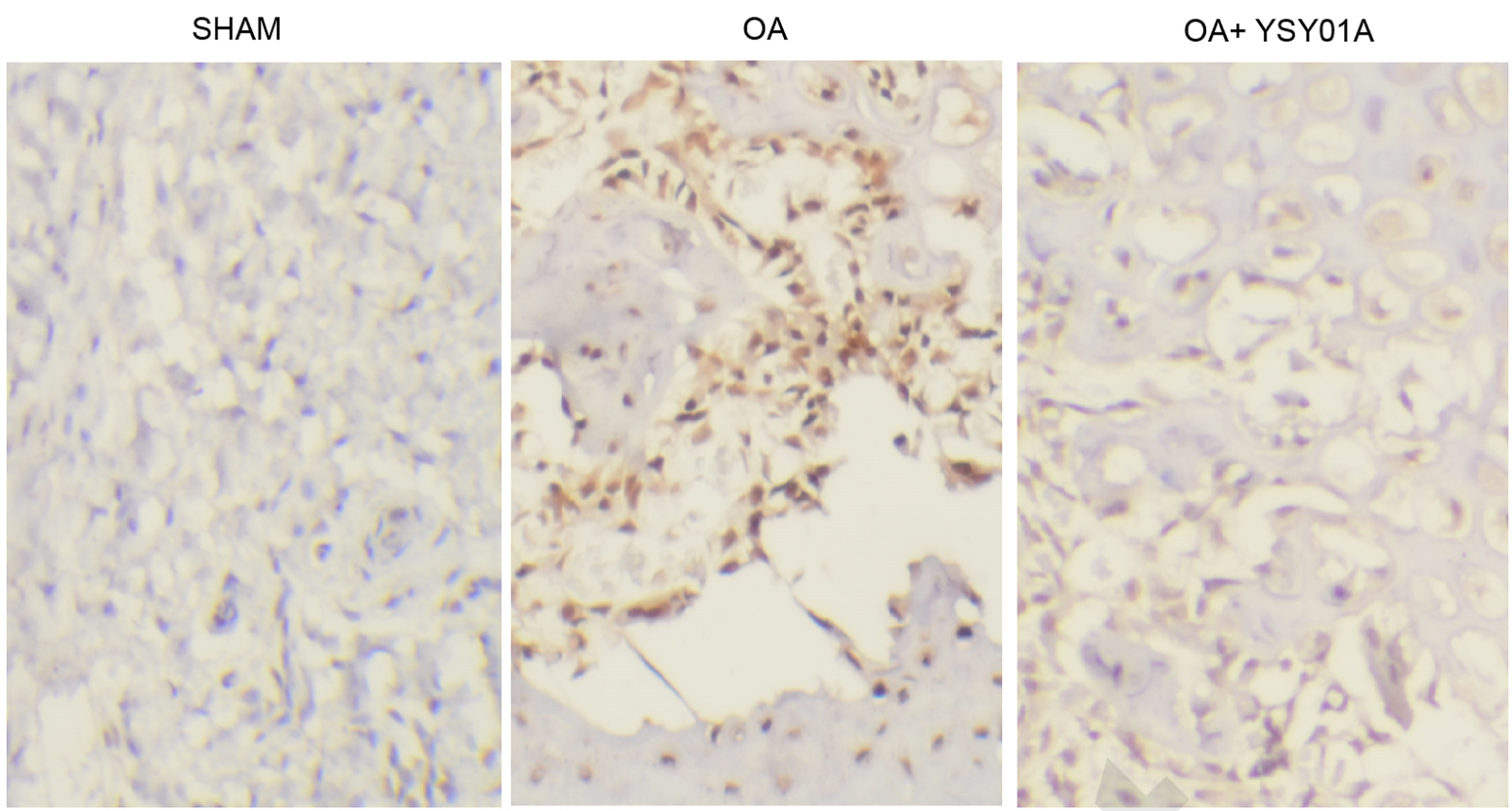

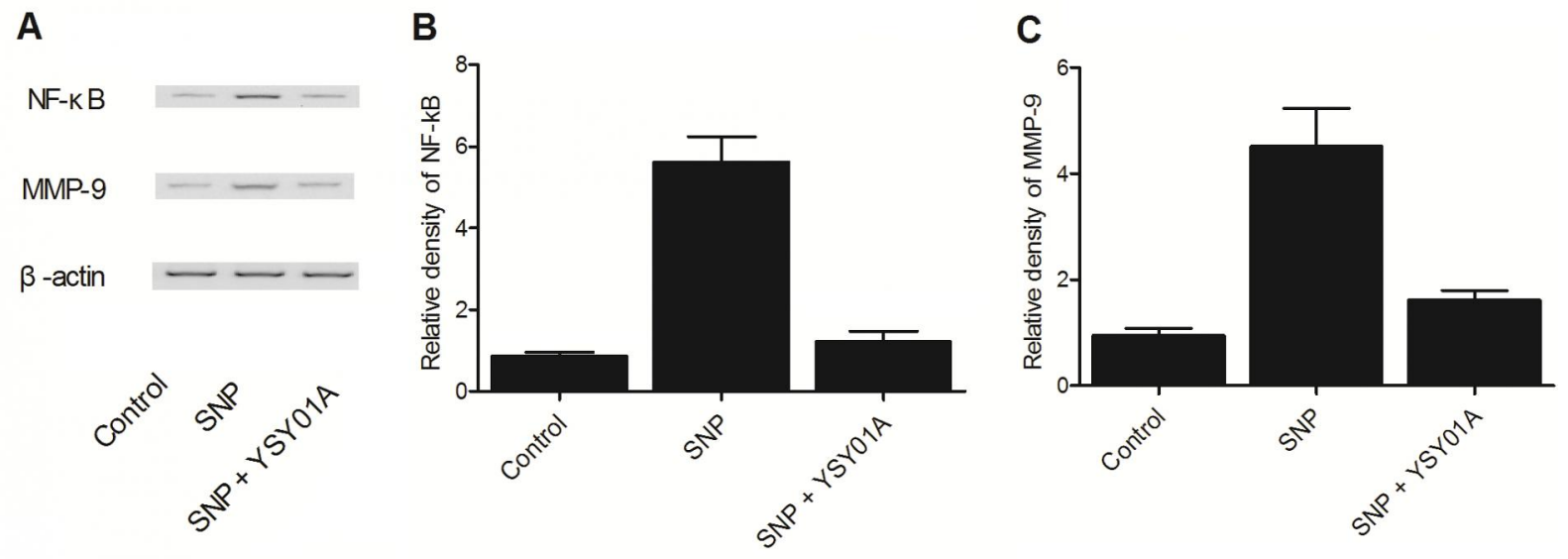

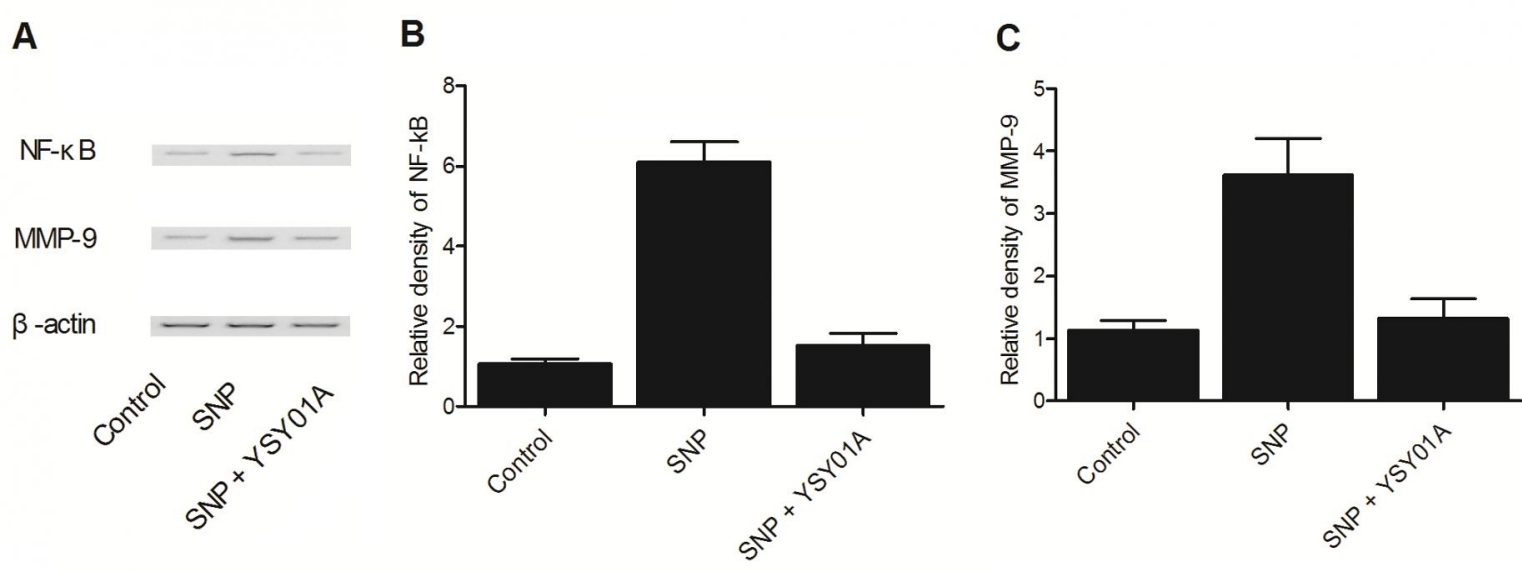

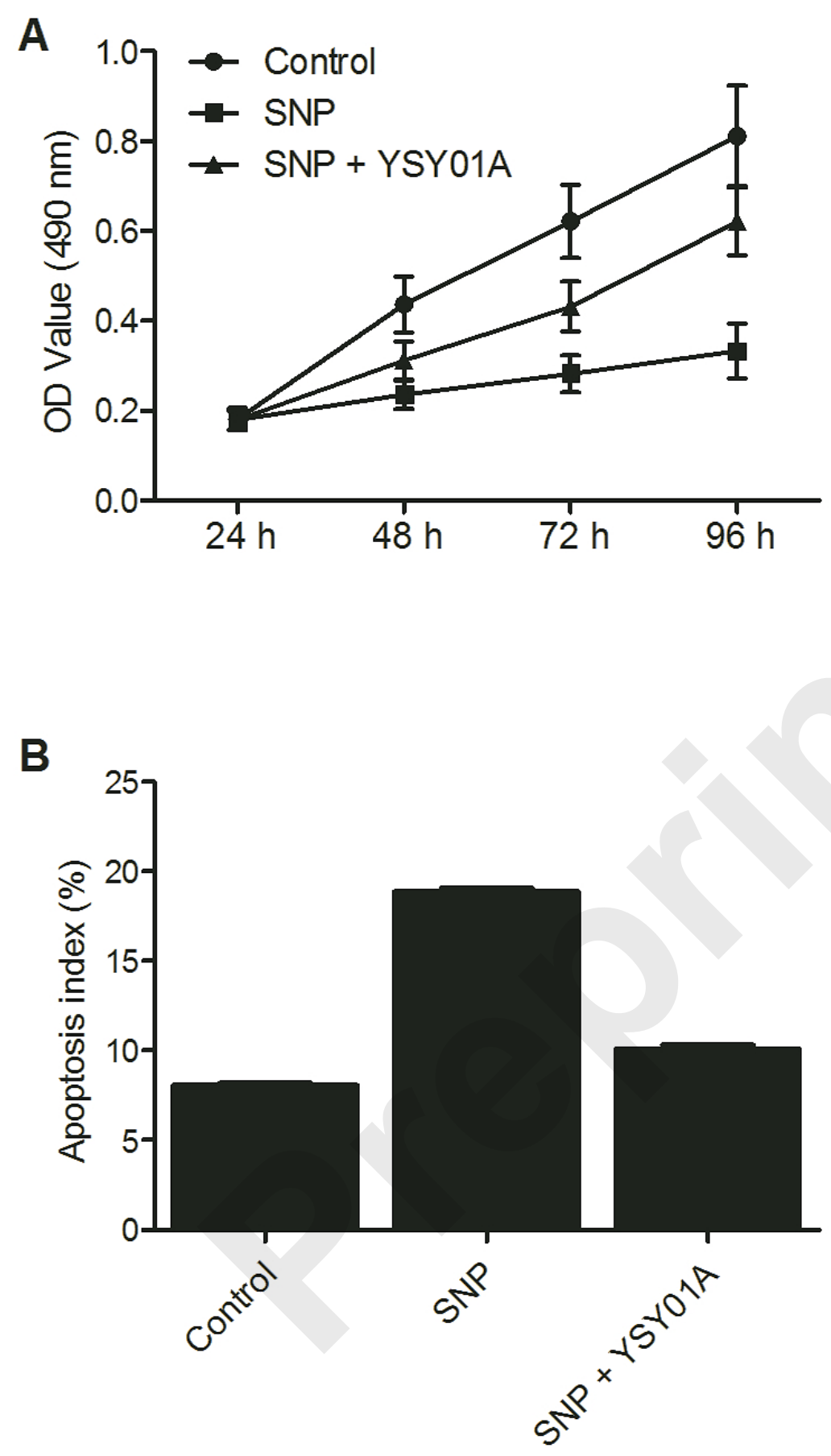

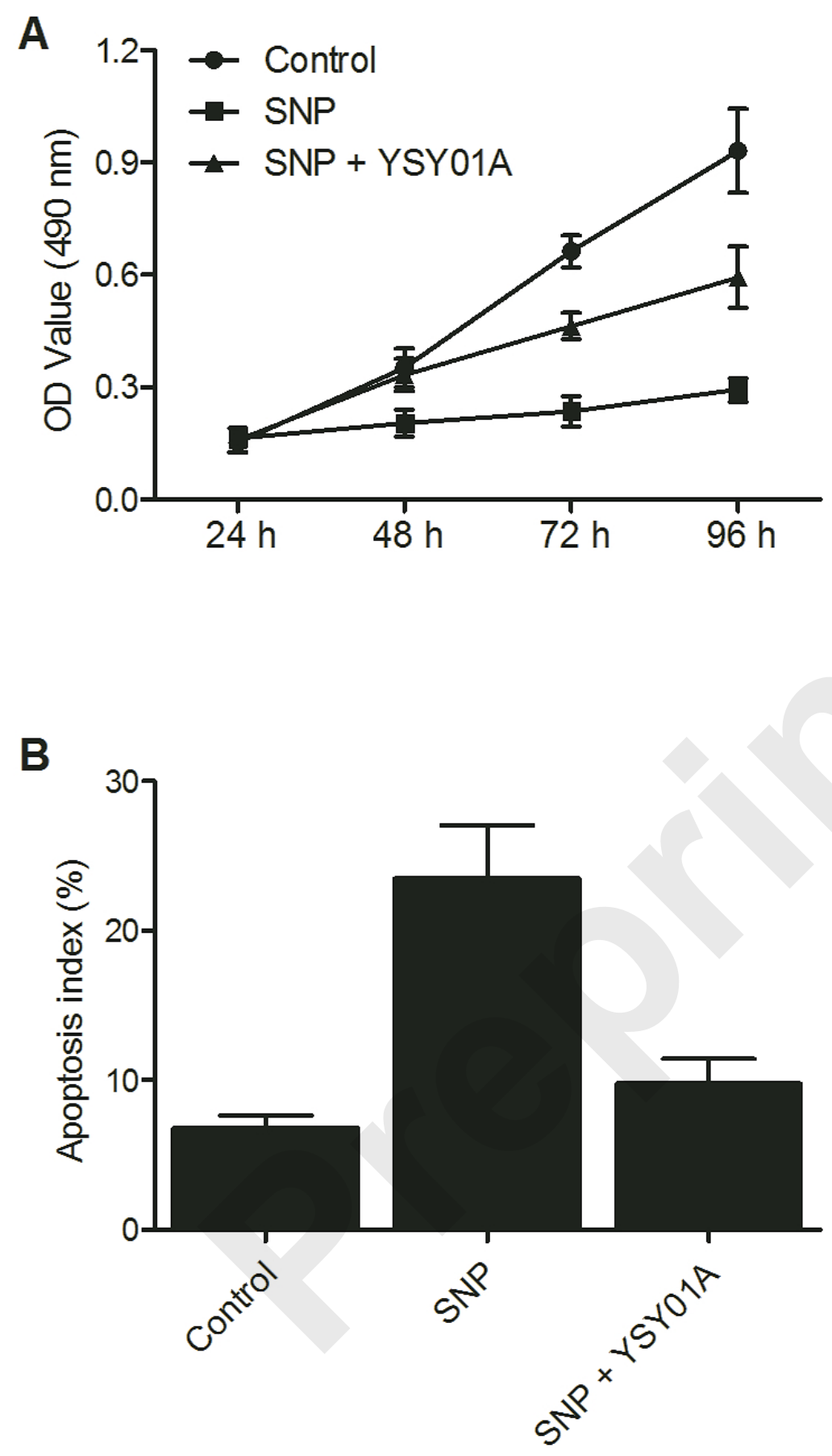